\title{
Biographies of Interviewees
}

\section{Frank Bolton}

b. 1924, Blackburn

RAF, acting sergeant and meteorologist

John Booker (pseud.)

b. 1920, Christchurch

Army, Royal Signals, Japanese translator

Richard Briar (pseud.)

b. 1922, Essex

Army, Ordnance Field Park, lance corporal

John Brierly (pseud.)

b. 1922, Sheffield

Army, Royal Signals, telecommunications mechanic

Frank Brown (pseud.)

b. 1920, London

RAF, armament engineer and flight sergeant

\section{Dennis Campbell (pseud.)}

b. 1924, Dundee

RAF, flight engineer

Lawrence Harney

b. 1926, Manchester

Royal Navy, ordinary seaman

\section{Jimmy Jacques}

b. 1920, St John's Wood

Army, Royal Artillery, gunner and projectionist

Francis Kennedy (pseud.)

b. 1919, Peckham

Army, Corps of Royal Engineers, mechanic 


\section{Albert Robinson}

b. 1912, West Kensington

Army, Catering Corps, cook

Stevie Rouse (pseud.)

b. 1921, London

WAAF, telephonist and corporal 